\title{
PHONOLOGY IN TEXT MESSAGES
}

\author{
MAŁgORZATA KUL \\ Adam Mickiewicz University, Poznań \\ kgosia@ifa.amu.edu.pl
}

\begin{abstract}
Text messages operate on a protocol which allows from 148 to 160 characters per message, including spaces between words. In such a highly circumscribed environment, writing is seriously hampered by the limited space and the usage of the numeric phone keypad. Thus, the advent of a new quality of the text language, sometimes referred to as "textese", was inevitable under those conditions. One of the characteristics of text messages is frequent deletion of letters in orthographical forms, like in the following example: IfYaMthWozNEBiGrUWdntHavNEFAcLft2Wsh (if your mouth was any bigger you wouldn't have anything else left to wash).

In order to investigate the nature of letter deletions in text messages a study was undertaken, which analyzed ten examples of text messages coming from various sources.

The aim of the study was to determine whether the deletion of letters was regular, the general prediction being that text messages are decoded via the mediation of their phonemic representations (or via mental reading). It was speculated that the regularities were governed by phonological principles such as the semiotic "figure and ground" principle (Dressler 1996) and the "rich-get-richer" principle (Donegan 1978/1985). The results demonstrate that phonology is very likely to govern reductions albeit without any recourse to the prosody level.More specifically, phonology apparently affects the pattern of deletions in text messages, whereas there is a marked tendency that stress assignment does not determine the nature of deletions.
\end{abstract}

KEYWORDS: Text messages; letter deletion; phonology; figure-and-ground principle; stress assignment.

A fictitious dialogue between contemporary Romeo and Juliet according to Sutherland (2002):

Rom: RUF2T? [Are you free to talk?]

Jul: OK [Make your move.]

Rom: Bf? [Do you have a boyfriend?]

Jul: No [Liar.]

Rom: CUA3 [I'll see you, any time, any place, anywhere.]

Jul: @club? [At the club?]

Rom: OK [Thinks. I'm on, "gr8 6 2nite" - great sex tonight!!!] 
1. Past research on the linguistic properties of text messages

It appears that text messages do not have an abundant linguistic literature, presumably due to the fact that text messages constitute a relatively recent communication medium. ${ }^{1}$ The sociological and discourse-related aspects of text messages seem to be extensively covered (Segerstad 2002; Bergs 2003; Thurlow 2003; Karwatowska and Szpyra-Kozłowska 2003; Bralczyk 2004; Kuźmiński 2004; Wolańska 2004; Malinowska 2004), whereas there is a marked tendency for the linguistic properties of text messages being merely signaled (Segerstad 2002; Thurlow 2003; Papen and Tusting 2006; Lopez Rua 2006).

Segerstad (2002) enumerates the following linguistic properties of short text messages:

omitting punctuation, unconventional punctuation, omitting blank space, spoken-like spelling, consonant writing, conventional abbreviations, unconventional abbreviations, either all capitals or all lower-case, exchange long words for shorter, emoticons, asterisks, symbol replacing word, punctuation

Thurlow (2003) lists the following:

(1) shortenings (i.e. missing end letters), contractions (i.e. missing middle let-

ters) and G-clippings and other clippings (i.e. dropping final letter)

(2) acronyms and initialisms

(3) letter/number homophones

(4) "misspellings" and typos

(5) non-conventional spellings

(6) accent stylizations

Papen and Tusting (2006) discuss contractions, abbreviations and consonant writing. Lopez Rua (2006) identifies various shortening devices in text messages such as clippings, phonetic respellings, capitalizations, letter homophones and number homophones.

The above linguistic properties highlight the most striking property of text messages, i.e. a shorthand manner of writing which, in turn, generates criticism of texting as a deviated communication mode. Bralczyk (2004) and Sutherland (2002) claim that texting fosters sloppy pronunciation habits and reduces language to a bare communicative minimum: "it is bleak, bald, sad shorthand. Drab shrinktalk [...] it masks dyslexia, poor spelling and mental laziness. Texting is penmanship for illiterates" (Sutherland 2002). Bralczyk (2004) discusses the texting habits in the Polish language and concludes that the influence of text messages is artificially inflated (Bralczyk 2004). Bralczyk (2004) and Sutherland (2002) fail to appreciate the productivity of texting and tend to underestimate its impact on everyday life and communication.

\footnotetext{
${ }^{1}$ Kotamraju (2004) has compiled an extensive bibliography on text messages.
} 


\section{The present study}

The stimulus of the study was an informal observation that in general text messages display an imperfectly realized orthography. In particular, letters are frequently deleted, as in the following examples: UTLKIN2ME ('are you talking to me') or $D o U$ BlveInLuv@1stSItOrShlIWlkByAgn ('do you believe in love at first sight or shall I walk by again'). As it has been mentioned above, a bibliographic search revealed that the literature on the subject of deletions in text messages could not be located, whereas discourse-oriented studies are abundant. As a result, a procedure had to be devised in the absence of relevant research which might provide guidelines on a method to study this new medium of communication in terms of deletions.

\subsection{Phonology-grounded predictions about the structure of text messages}

It is be assumed for the sake of the present study that text messages are decoded via the mediation of their phonemic representation, as it seems to be the case with the representation of the word night as nite. In other words, the issue which the present paper endeavors to address is the mental reading by their recipients of the reconstructed, phonemic representations of imperfectly realized text messages, in which phonological principles are expected to govern the deletion of segments. ${ }^{2}$

One of the phonological principles which might contribute to deletions is the semiotic principle of "figure and ground" (Dressler 1996). ${ }^{3}$ The principle "predicts that figures tend to be foregrounded, grounds to be further backgrounded" (Dressler 1996: 42). Since in phonology, consonants (C) are figures and vowels (V) are grounds, consonants are likely to be preserved, whereas vowels are likely to be deleted. Whenever consonant clusters occur, these tend to be reduced, since $\mathrm{CV}$ is the optimal realization of the figure and ground contrast, better than $\mathrm{CCV}$ or $\mathrm{CVC}$.

Another principle governing phonology is the "rich-gets-richer" principle developed by Donegan (1978/1985), which specifies the precise conditions for Dressler's (1996) figure and ground principle. ${ }^{4}$ Donegan $(1978 / 1985)$ argues that figures which appear in strong positions are preserved or strengthened (e.g. consonants word initially or initially in stressed syllables are preserved, or consonants are strengthened by the process of stopping), whereas grounds which appear in weak positions are weakened

\footnotetext{
${ }^{2}$ Text messages were reconstructed rather than intended, since there is no access to intentions.

${ }^{3}$ A well-known example coming from gestalt psychology is the picture representing either two faces or a vase, depending on what the viewer considers to be the grounds or the figures.

${ }^{4}$ The difference between the two principles is quite subtle. In fact, the "rich-gets-richer" principle is not a separate principle but rather should be viewed as a development of the "figure and ground" principle. The former principle extends the latter one in terms of weak/strong positions.
} 
(e.g. vowels in unstressed position undergo reduction to schwa or zero, consonants in word final positions are deleted). ${ }^{5}$

The deletions found in text messages are expected to correspond to reductions in speech (cf. the mental nature pf phonological processes, Stampe 1973/1979). In text messages, the letters which represent vowels in unstressed positions should be deleted within the domain of the weakly accented syllable. Symbols of consonants are not predicted to be subject to elision. Therefore, the following predictions about the behavior of text messages were made:

\section{Prediction 1}

Consonant letters are not likely to be deleted, especially word initially or initially in stressed syllables.

Prediction 2

Vowel letters in unstressed positions are likely to be deleted.

If these predictions were verified, an influence of phonology on text messages could be declared.

\subsection{The corpus}

The original corpus included 50 text messages where the length of individual text messages ranged from a few words to a few sentences. For the purpose of the study a sample of 10 messages was selected, total number of word tokens in these ten selected text messages being 96 , out of which 54 word tokens underwent reductions. ${ }^{6}$ Consequently, the study analyzes letter deletions in the 54 word tokens. In the light of the modest data sample, the study remains only a qualitative case study as the quantitative information in the paper is reduced to size.

Words such as you which were represented as $U$ or two as 2 or at as @ (UTLKIN2ME orDoUBlveInLuv@1stSItOrShlIWlkByAgn)were excluded from the study as they employ the metaphonological phenomenon of graphemic-phonemic manipulation (undoubtedly phonologically grounded) as well as they require an awareness of homonymy. Besides, the issue of metaphonological devices was separately addressed (Kul 2007). Since the study attempts to determine whether letter deletions are governed by phonology, metaphonological phenomena are not taken into account in the present paper.

Most of the text messages analyzed in the study came from the website www.transl8it.com, while other examples came from online or press articles. In terms

\footnotetext{
${ }^{5}$ Foregrounding (Verdeutlichung) processes have the clarification function, whereas the backgrounding ones (Entdeutlichung) serve the obscuration function.

${ }^{6}$ In the study, words were used in the sense of tokens, not types.
} 
of data selection, text messages were thematically restricted to boy-girl kind of exchanges. Matchmaking-related text messages appeared to be the most frequent on the web as there exists a number of ready-made chat-up lines in the form of text messages, obtained via paid or free servers. Thus, frequency and availability justify the selection of particular corpus. It must be noted, however, that the results obtained in the study are valid only for romance-oriented SMS users and may vary according to various user groups.

\subsection{Length}

For the sake of the study, the selected text messages were divided into "units" constituting meaningful entities. The messages in the study differed in length ranging from four to thirteen words.

The compilation of a small corpus for the study presented methodological difficulties. $^{7}$ The question arose of what textual unit should be selected for a systematic analysis. Particular words, though sometimes very interesting themselves, would not suffice, whereas the sentence level was also considered inappropriate since the length of text messages varied greatly. The underlying principle in selecting a unit was prosody, with the view of conducting further research such as addressing the issue of the behavior of text messages within the domain of phonological phrase.

\subsection{The procedure}

In order to determine the extent of the phonological information in the orthographic form, the full, reconstructed representation was compared with the text message version. There were two angles from which text messages were studied, namely the deleted and retained elements. For this purpose, the word tokens were analyzed in the form of tables, like in the following example: Talking vs. $T l k i n$.

The point is that silent letters, or letters representing vowels in speech, such as $/ 1 /$ in the above example, were treated as consonants for the sake of the study due to the fact that the visual properties of orthography cannot be neglected in the written forms. Moreover, if the silent /1/ were removed, the word might be misinterpreted as taking. Accordingly, /g/ was treated not as a part of the digraph but as a separate consonant due to the fact that one of the digraph elements is more likely to carry the phonological in-

\footnotetext{
${ }^{7}$ The discussion of MLU serves as an illustration of the fact that choosing a unit for the sake of analysis is an intricate issue. MLU was introduced by Brown (1973) in order to capture the relation between sentence length and a child's syntactical development. The growing length of utterances is expected to reflect the incorporation of subsequent portions of linguistic knowledge by a child. MLU was followed by the guidelines on calculating the mean length of utterances.
} 
formation about the sound it denotes (here $/ \mathrm{n} / \mathrm{seems}$ to be superior in conveying the nasal gesture). The treatment of silent letters as consonants allowed taking into account the knowledge of phonotactics which certainly influences decoding of the written form.

Two parameters were taken into account: global and local. The global one considered the word class, whereas the local one was further subdivided into the position in a word for consonants and stress assignment for vowels. In terms of stress, the lexical stress was taken into account.

\section{Results}

The results are not presented against a fixed number such as the total number of word tokens in the corpus as the study employs more than one parameter. Rather, the absolute numbers are calculated against the total number relevant for a given calculation, for instance, either the total number of deleted vowels or the total number of deleted consonants. The size of the corpus justifies the use of absolute numbers as the use of percentages would deceptively suggest general results.

\subsection{The extent of reduction}

Table 1. The extent of reduction

\begin{tabular}{ll}
\hline Total number of word tokens & 96 \\
Reduced word tokens & 54 \\
\hline
\end{tabular}

Table 1 shows the proportion of reduced to unreduced words within the corpus which permits to demonstrate the extent of reduction. The data would seem to suggest that, contrary to the expectations, text messages do not seem to display massive reductions. As shown in Table 1, 54 words from the corpus are reduced. Examples: man, me, is, in (unreduced words), vry, $k n w, l v$ (reduced words).

\subsection{Proportion of deleted vowels to consonants}

Table 2. Proportion of deleted vowels to consonants

\begin{tabular}{ll}
\hline Deleted vowels & 9 \\
Deleted consonants & 1 \\
\hline
\end{tabular}


The values in Table 2 are given in numbers where the two variables are compared against each other. Table 2 shows that as many as 59 vowels were deleted, whereas only 21 consonants were subject to deletion. The data shown in Table 2 also illustrate the fact that vowels are more likely to be deleted: more than twice as many vowels have been deleted in comparison to consonants. The next step is to examine the stress value of the deleted vowels and the position of the deleted consonants as it was indicated by the local parameters.

\subsection{Local parameters}

3.3.1. Stress assignment of the deleted vowels

Table 3. Stress of the deleted vowels

\begin{tabular}{ll}
\hline Deleted vowels in monosyllabic words & 42 \\
& \\
Deleted vowels in polysyllabic words \\
(stress of the deleted vowels) \\
Deleted stressed vowel 1 & 7 \\
Deleted stressed vowel 2 & 5 \\
Deleted stressed vowel 3 & 0 \\
Deleted unstressed vowel 1 & 1 \\
Deleted unstressed vowel 2 & 2 \\
Deleted unstressed vowel 3 & 2 \\
\hline
\end{tabular}

Table 3 shows the distribution of stress in the deleted vowels. In polysyllabic words, phonotactics was employed and consequently, the vowels are numbered as: Vowel 1 if it was the vowel of the first syllable, Vowel 2 if it was the vowel of the second syllable and Vowel 3 if it was the vowel of the third syllable. The numbers are calculated against the total number of the deleted vowels in the corpus. One can see that deleted vowels in monosyllabic words constitute as much as 42 of the deleted vowels ( frm, hrt, mst). The vowels deleted in stressed positions account for 7 and 5 in stressed V1 and V2 positions accordingly, whereas no single third vowel is dropped. Thus, Table 3 provides powerful evidence that in text messages vowel deletion is the domain of vowels in monosyllabic words. 


\subsubsection{Position of the retained consonants}

Table 4. Position of the retained consonants in polysyllabic words

\begin{tabular}{ll}
\hline Initial & 13 \\
Medial & 13 \\
Final & 14 \\
\hline
\end{tabular}

Table 4 shows the retained consonants in polysyllabic words and specifies if they were initial, medial or final with reference to the local parameter of the position of the consonant in the word. The numbers are calculated against the total number of the retained consonants in the corpus. All consonants, regardless of the position on a word, were retained at an equal level. Examples: yaslf, posesn, tlkn.

Table 5. Position of the retained consonants in monosyllabic words

\begin{tabular}{ll}
\hline Initial & 38 \\
Final & 38 \\
\hline
\end{tabular}

Table 5 shows the retained consonants in monosyllabic words and specifies if they were initial or final with reference to the local parameter of the position of the consonant in the word. The numbers are calculated against the total number of the retained consonants in the corpus. All consonants, regardless of the position on a word, were retained at the level of 38. Examples: mst, pls, wif.

The data presented in Tables 3, 4 and 5 are now investigated from the point of view of the two predictions. This helps to systematize the results for vowels and consonants.

(a) Prediction 1 refers to the figure and ground principle, according to which consonants are likely to be retained and vowels are likely to be deleted. As it can be seen in Tables 2 and 4, consonants are deleted to a very small extent (21 consonants), whereas vowels are dropped twice that often (59 vowels). The semiotic figure and ground principle, which expects consonants to be the retained figures and vowels to be the deleted grounds, is verified. Moreover, not only are the initial and final consonants retained, but so are middle consonants (Table 4). Therefore, Prediction 1 holds true for text messages.

(b) Prediction 2 refers to the rich-gets-richer principle, according to which vowels are expected to undergo reduction to schwa in unstressed positions. The inspec- 
tion of results presented in Table 3 shows that in text messages vowels are deleted in stressed positions. This seems to run counter to the second prediction since the results for deleted vowels do not seem to corroborate the prediction number two. Moreover, prediction 2 also stipulates that consonants in weak positions are likely to be deleted and in strong ones are likely to be preserved (i.e. word initially or initially in stressed syllables). Table 4 illustrates that in word initial position consonants are preserved, whereas in word final position they are preserved as well. Remarkably, most of polysyllabic words in the corpus were trochees, and as a result, the tendency for trochees to preserve consonants word finally in unstressed position fails to support Prediction $2 .{ }^{8}$ Therefore, Prediction 2 is not verified by the results obtained in the study.

The consonantal frame of words is retained fully, which agrees with the psycholinguistic research on the lexicon. The lexical search and processing theories stipulate the importance of 'frames' which are the beginning and the end of a word. If the frames exist, processing is possible even if the middle part is incomplete or misplaced. In analyzed instances of text messages, neither the initial and final consonants were deleted (Tables 4 and 5). The existence of frames for a word is known as "the bathtub effect" in psycholinguistics. ${ }^{9}$

Summing up, letter deletions in text messages seem to fail to behave according to the "rich-gets-richer" principle; however, they apparently observe the "figure-andground" principle. Furthermore, from the facts it is possible to infer that the linguistic behavior of consonants is in accordance with the bathtub effect.

\subsection{Global parameter}

The global parameter considers the word class which traditionally is referred to as parts of speech (Greenbaum 1989). Typologically, they can be divided into open and closed word classes: "open classes are readily open to new words; closed classes are limited classes that rarely admit new words" (Greenbaum 1989: 104). The members of the open class are nouns, adjectives, main verbs and adverbs, whereas pronouns, determiners, auxiliaries, conjunctions and prepositions constitute the closed class.

\footnotetext{
${ }^{8}$ Statistically speaking, among the 13 polisyllabic words there were two tri-syllabic examples (anything, possession), three iambic examples (again, believe, yourself), whereas eight were trochees (bigger, heaven).

${ }^{9}$ The idea is that the particular position of a body in a bathtub makes head and feet the most prominent and head even more than feet. This applies to memory for words. "People remember the beginnings and ends of words better than the middles, as if the word were a person lying in a bathtub, with their head out of the water one end and their feet out the other. And, just as in a bathtub the head is further out of the water and more prominent than the feet, so the beginnings of the words are on average better remembered than the ends [...] people tend to recall the beginnings and ends of words they cannot otherwise remember " (Aitchinson 1987: 119).
} 
Radford (2004) classifies the word categories along morphological and syntactical criteria, while in problematic cases, a substitution test is performed which consists in "seeing whether (in a given sentence) the word in question can be substituted by a regular noun, verb, preposition, adjective or adverb etc." (Radford 2004: 33). Nouns, verbs, prepositions, adjectives and adverbs are referred to as content words (contentives) which means that they have substantive descriptive content. The other categories, namely particles, auxiliaries, pronouns, complementizers and determiners belong to function words (functors) which determine the grammatical properties, such as case, gender, person, number: "contentives have substantive lexical content (i.e. idiosyncratic, descriptive content which varies from one lexical item/word to another, whereas functors have functional content" (Radford 2004: 35).

According to Longman Grammar of Spoken and Written English (1999), class membership is not a clear-cut case as the degree determines the membership of a particular category. Notwithstanding, lexical words, function words and inserts are the three basic classes of words. Lexical words are "the main building blocks of text" (Longman Grammar of Spoken and Written English 1999: 55) and carry the main meaning: nouns, verbs, adjectives and adverbs which refer not only to specific and concrete entities but also to abstract concepts, states and qualities. Their number is high and they are usually stressed. The results of a corpus search established that the information load of lexical words varies across registers, moreover, lexical words have a different lexical density in spoken or written texts.

Function words have high frequency and are described as short and unstressed elements of speech which have no lexical meaning. Inserts convey emotions as well as trigger interaction, thus it can be stated that they play a marginal role in comparison with lexical and function words.

The two classes have different properties. Function words are repeatable, not "special" as content words are (in particular in richly inflected languages). There are not many of them, but they carry the relations between main categories since prepositions, unlike verbs, determine the semantic difference between phrasal verbs. Main categories are one of the kind, they convey the main information since the noun moon is different from the noun flower.

Longman Grammar of Spoken and Written English (1999) contains distributional statements based on corpus findings: for example, nouns are the most frequent words, on average every fourth word is a noun, whereas verbs occur every tenth word. The distribution of lexical words, function words and inserts (in two text samples) is shown in Table 5 .

The global parameter prediction refers to the stress assignment: according to Longman Grammar of Spoken and Written English (1999), lexical words are stressed, whereas function words are unstressed. This means that function words are predicted to undergo reductions, whereas lexical words are not likely to drop letters. Moreover, function words are predictable as opposed to the lexical ones since function words do not carry the main meaning like lexical words do. Since Prediction 1 and Prediction 2 
Table 5. The distribution of lexical words, function words and inserts in conversation and news (after Longman Grammar of Spoken and Written English (1999: 61)

\begin{tabular}{lcc}
\hline & Conversation & News \\
\hline Lexical words & 41 & 63 \\
Function words & 44 & 37 \\
Inserts & 15 & - \\
\hline
\end{tabular}

referred to the local parameter (stress assignment), the global parameter, i.e. word classes, allows to formulate Prediction 3. Therefore, the following prediction about the behavior of text messages in terms of the global parameter was made:

Prediction 3

Function words are likely to delete letters due to their lesser significance, whereas lexical words are not.

Table 6: Reduction across word classes

\begin{tabular}{lc}
\hline Function words & 6 \\
Auxiliaries & 2 \\
Pronouns & 1 \\
Question words & 2 \\
Conjunctions & 1 \\
Intensifiers & 2 \\
Quantifiers & 1 \\
Determiners & 15 \\
\hline Total (function words) & \\
\hline & \\
\hline Lexical words & 15 \\
Verbs & 11 \\
Nouns & 7 \\
Adjectives & 2 \\
Prepositions & 2 \\
Adverbials & 37 \\
\hline Total (lexical words) & 2 \\
\hline
\end{tabular}


Table 6 illustrates the occurrence of letter deletions across word classes. The numbers are calculated against the total number of the deleted words in the corpus. One can see that lexical words - verbs, nouns and adjectives - are reduced most frequently: 15 (examples: $h r t, w l k, l v), 11$ (examples: $m t h$, $h v n$, thng) and 7 (examples: bigr, sor, sngl) respectively. The number of reduced function words is much lower, revolving around 2-1 instances for pronouns, prepositions etc., with the notable exception of auxiliaries. The results obtained with the global parameter show that function words were not the most reduced words, thus, parameter 3 is not verified by text messages.

It is worth comparing the data extracted from the text messages in the form of Table 6 against Table 5. The data from Table 5 establish the primacy of lexical words over function words in terms of frequency. Table 6 is therefore in accordance with the distribution statements as far as written texts are concerned. Regardless of word class and semantic content, the words most frequently occurring in the text messages are subject to the greatest reductions. This observation lends support to the frequency-based theories in phonology (Bybee 2001).

\section{Conclusions and further research}

The study aimed at preliminary analysis of the linguistic properties in text messages. Therefore, the results offer a tentative explanation of the influence of phonology on the medium.

First, the results show that phonology apparently affects the pattern of deletions in text messages. The semiotic figure-and-ground principle (Dressler 1996) is in force since consonants are likely to be preserved and vowels are likely to be deleted, whereas final consonants in unstressed position are likely to be preserved. Deletions in text messages fail to observe the rich-gets-richer principle (Donegan 1978/1985), thus contrary to Prediction 2, vowels are deleted in stressed positions. This means that there seem to be no obvious effect of prosody. Stress assignment is not likely to determine the nature of deletions. Second, text messages appear to reduce lexical words rather than function words, contrary to Prediction 3. Third, the pattern for consonants seems to corroborate the "bathtub effect" (there was no such prediction, the third conclusion emerged in the course of investigation).

Further research could take up the problems which were not addressed by the present study. First, a larger corpus would allow gaining a further insight into the nature of deletions. Since the results of this study are based on a small sample size, certain caution should be taken when making any generalized conclusions. Therefore, the described analysis will be expanded to a bigger database. Beside more detailed further work on the issue of deletions may take into consideration gathering of data from other languages. Next, the question of psycholinguistic representation of the lexicon could be addressed. The study demonstrated that coding of representations proceeds along with phonology, and the issue of silent letters carrying the functional load calls for more research as the texter must make a decision which letter should be deleted. Finally, the 
constraints on deletions (how much, which classes of sounds) will constitute another angle from which deletions in text messages can be viewed.

\section{REFERENCES}

Aitchinson, J. 1987. Words in the mind. An introduction to the mental lexicon. Oxford: Blackwell.

Bergs, A. 2003. "The linguistics of text messaging”. Paper presented at the XVIth International Conference on Historical Linguistics ICHL 2003, Copenhagen, 11th-15th August 2003. $<$ http://www.hum.ku.dk/ichl2003/abstracts/section10.html> Retreived on 23 May 2004.

Bralczyk, J. 2004. "HALO, HALO! ZYCZE CI WESOLYCH SWIAT CALUJE I 3MAJ SIE :)". Idea Mobile.

$<$ http://www.idea.pl/portal/map/map/ideamobile?type=IDEA_MOBILE $>$ Retrieved 28 Jun 2004.

Bybee, J. 2001. Phonology and language use. Cambridge: Cambridge University Press.

Donegan, P.J. 1978 [1985]. On the natural phonology of vowels. New York: Garland.

Dressler, W.U. 1996. "Principles of naturalness in phonology and across components". In Hurch, B. and R.A. Rhodes (eds.), Natural phonology: The state of the art. Berlin: Mouton de Gruyter. 41-52.

Dziubalska-Kołaczyk, K. 2001. "Phonotactic constraints are preferences". In DziubalskaKołaczyk, K. (ed.), Constraints and preferences. (Trends in Linguistics. Studies and Monographs 134.) Berlin: Mouton de Gruyter. 69-100.

Ellison, S. 2001. “UTLKIN2ME? Can U speak cell phone?” The Wall Street Journal Online. $<$ http://zdnet.com.com/2100-11-529548.html> Retrieved 16 Jan 2004.

Gentle, P. 2003. "R U an SMS poet?” The World of English 1/2003(64).

Greenbaum, S. 1989. A college grammar of English. London: Longman.

Härmä, J., J. Korhonen and T. Nevalainen (eds.). 2006. Neuphililogische Mitteilungen. (Bulletin of the Modern Language Society, 2 CVI 2005.) Helsinki: Modern Language Society.

[no author] [nd] "Investigating the language of new communication technologies". $<$ http://www.netting-it.com> Retrieved 5 Jan 2004.

Karwatowska, M. and J. Szpyra-Kozłowska. 2003. "Dowcip i wulgarność - cechy rozmów uczniowskich prowadzonych za pośrednictwem SMS-ów" [Humor and vulgarity - the properties of SMS-mediated students' interactions]. Dialog a nowe media [Dialogue and the new media]. Druga Internetowa Konferencja Naukowa [2nd Online Scientific Conference]. University of Silesia, Katowice, Poland, March-April 2003.

$<$ http://uranos.cto.us.edu.pl/ dialog/archiwum/referaty.html> Retrieved 22 May 2004.

Kotamraju, N.P. 2005. "Mobile phone/SMS/instant messaging research". $<$ http://socrates.berkeley.edu/ nalinik/mobile.html> Retrieved 16 Jan 2004.

Kul, M. 2007. "Metaphonology in text messages". In Lewandowska-Tomaszczyk, B., T. Płudowski and D. Valencia Tanno (eds.). 429-442.

Kuźmiński, M. 2004. "SMS: niesforne dziecko ery mediów” [SMS: The wild child of the media era]. Mediator 3(4). 14-22.

Lewandowska-Tomaszczyk, B., T. Płudowski and D. Valencia Tanno (eds.). 2007. The media and international communication. (MediaCom 2005.) Frankfurt am Main: Peter Lang.

Longman Grammar of Spoken and Written English. 1999. Harlow: Pearson Education Limited.

Lopez Rua, P. 2006. "Shortening devices in text messaging: a multilingual approach". In Härmä, J., J. Korhonen and T. Nevalainen (eds.). 139-155. 
Malinowska, E. 2004. "Napisz do mnie przez telefon - od epistoły do SMS-a". In Ostaszewska, D. (ed.). 189-197.

Maybin, J. and J. Swann (eds.). 2006. The art of English: Everyday creativity. Glasgow: Palgrave Macmillan.

Ostaszewska D. (ed.). 2004. Gatunki mowy i ich ewolucja [Speech genres and their evolution]. (Vol. 2: Tekst a gatunek [Text and genre].) Katowice: Wydawnictwo Uniwersytetu Śląskiego.

Papen, U. and K. Tusting. 2006. "Literacies, collaboration and context". In Maybin, J. and J. Swann (eds.). 312-359.

Radford, A. 2004: English syntax: An introduction. Cambridge: Cambridge University Press.

Segerstad, Y.H. af. 2002. Use and adaptation of written language to the conditions of ComputerMediated Communication. [Unpublished PhD dissertation, Göteborg University.]

$<$ http://www.ling.gu.se/\%7Eylva/Dokument/ylva_diss.pdf $>$ Retrieved 8 Jun 2004.

Stampe, D. 1973 [1979]. A dissertation on natural phonology. New York and London: Garland Publishing, Inc.

Sutherland, J. 2002. "Cn u txt?” The Guardian, 11 Nov 2002.

$<$ http://www.guardian.co.uk/print/0,3858,4543918-103680,00.html> Retrieved 16 Jan 2004.

The Text 2nite Home Page. [nd]. "Funny text messages". $<$ http://www.txt2nite.com/menu.html> Retrieved 6 Jan 2004.

The T Mobile Home Page. [nd]. "Shorthand dictionary". $<$ http://www.t-mobile.com/messaging/default.asp?nav> Retrieved 6 Jan 2004.

Thurlow, C. 2003. "Generation txt? The sociolinguistics of young people's text-messaging". Discourse Analysis Online 2003, vol. 1, no. 1.

$<$ http://extra.shu.ac.uk/daol/previous/v1_n1.html> Retrieved 16 Jan 2004.

Wolańska, E. 2004. Krótka wiadomość tekstowa (SMS) jako proces i struktura. Właściwości komunikacyjne, tekstowe $i$ stylistyczne [The short text message (SMS) as a process and a structure. The textual and stylistic properties.] [Unpublished PhD dissertation, Warsaw University.].

<http://bazy.opi.org.pl/raporty/opisy/synaba/119000/sn119536.htm> Retrieved 2 Jan 2006. 


\section{APPENDIX}

The text messages analyzed in the study.

(1) UTLKIN2ME - '(Are) you talking to me?'

(2) DdUHrtYaslfWenUFeLFrmHvn? - 'Did you hurt yourself when you fell from heaven?'

(3) WAN2CAPIC? - '(Do) you want to see a picture?'

(4) TonlyThngThtLOksGOdOnMeIsU - 'The only thing that looks good on me is you.'

(5)DoUBlveInLuv@1stSItOrShlIWlkByAgn - 'Do you believe in love at first sight or shall I walk by again?'

(6) IfYaMthWozNEBiGrUWdntHavNEFAcLft 2 Wsh. - 'If your mouth was any bigger you wouldn't have anything else left to wash.'

(7) a Sngl Man In PoSeSn Of A GOd 4tun, Mst B In 1nt Of A WIf. - 'A single man in possession of good fortune must be in want of a wife.'

(8) 1 wld like 2 wsh u all a Mry xmas nd a vry hapy nu yr - 'One would like to wish you all a Merry Christmas and a very Happy New Year.'

(9) dis dA nxt wk ul b sorE dat u faut wit me! so dare! ha! - 'This day next week you'll be sorry that you fought with me! So dare! Ha!'

(10) $i$ nd sum 12 lv me $n$ care 4, if ur dat 1 pls lt me knw 2nite cuz $i$ cnt w8 any mr - 'I need someone to love me and care for. If you are that one please let me know tonight because I can't wait any more.' 